\title{
Identification of Methicillin-Resistant Staphylococcus Epidermidis (MRSE) in Mild, Moderate and Severe Acne Patients
}

\author{
Farida Tabri ${ }^{1}$, Nasrum Massi ${ }^{2}$, Risalinda Sjahrir ${ }^{2}$, Ilhamjaya Patellongi ${ }^{3}$, Siswanto Wahab ${ }^{1}$, \\ Khairuddin Djawad ${ }^{1}$ \\ ${ }^{1}$ Department of Dermatology and Venereology, Faculty of Medicine, Hasanuddin University, Makassar, Indonesia \\ ${ }^{2}$ Department of Medical Microbiology, Faculty of Medicine, Hasanuddin University, Makassar, Indonesia \\ ${ }^{3}$ Department of Physiology, Faculty of Public Health, Hasanuddin University, Makassar, Indonesia
}

\section{Email address:}

farida_tabri@yahoo.com (F. Tabri)

\section{To cite this article:}

Farida Tabri, Nasrum Massi, Risalinda Sjahrir, Ilhamjaya Patellongi, Siswanto Wahab, Khairuddin Djawad. Identification of MethicillinResistant Staphylococcus Epidermidis (MRSE) in Mild, Moderate and Severe Acne Patients. American Journal of Clinical and Experimental Medicine. Vol. 5, No. 1, 2017, pp. 5-9. doi: 10.11648/j.ajcem.20170501.12

Received: December 9, 2016; Accepted: December 21, 2016; Published: January 16, 2017

\begin{abstract}
Acne cases caused by Staphylococcus epidermidis bacteria have been reported previously. This study identified methicillin resistant Staphylococcus epidermidis (MRSE) directly from pus speciment from acne patients at various degree of severity. There were $61 \%$ positive samples detected by Polymerase Chain Reaction (PCR) for MRSE using target gene mecA for PCR amplification of acne samples. Significant correlation between the severity of acne and its causative agent (MRSE) was not found.
\end{abstract}

Keywords: Staphylococcus epidermidis, Acne Level of Severity, mecA-PCR

\section{Introduction}

Humans are colonized by many different staphylococcal species. Members of the genus Staphylococcus are common colonizers of the skin. Two main groups are distinguished by their ability to coagulate blood: coagulase-positive Staphylococci, with the most important species being Staphylococcus aureus, and coagulase-negative staphylococci, which comprise most species including Staphylococcus epidermidis. The largest density of staphylococci is found in sweat glands and on mucous membranes surrounding body openings [1].

While $S$. epidermidis is commensal on the skin, it is considered a pathogen if it breaches the skin surface and enters the bloodstream [2]. S. epidermidis is the staphylococcal species that is most frequently isolated from the human skin. It predominantly colonizes the nose, axillae and the head [1]. S. epidermidis, which are normal inhabitants of the skin, were isolated from acne vulgaris inflammatory sites of patients [3].
Acne vulgaris is a chronic inflammatory disorder of pilosebaceous follicles that affects more than 85 percent of adolescents and young adults. Four major factors are involved in the pathogenesis including increased sebum production, hypercornification of the pilosebaceous duct, an abnormality of the microbial flora especially colonization of the duct, and the production of inflammation. Several factors that may influence acne include diet, menstruation cycle, sweating, stress, ultra violet radiation and occupation [4].

An acne lesion, particularly a closed comedone or deepseated abscess in an open comedone, creates an anaerobic microenvironment which facilitates overgrowth of Propionibacterium acnes ( $P$. acnes). It has been reported that $P$. acnes, $S$. epidermidis, and other skin microflora co-exist in acne lesions [5]. Although acne is not considered an infectious disease, three major organisms were isolated from the surface of the skin and the pilosebaceous duct of patients with acne including $P$. acne, S. epidermidis and Malasezia furfur. Depending on the severity of the disease, acne patients receive topical or systemic antibiotic therapy, or a combination [4]. 
Antibiotic resistance is frequent in many staphylococci and significantly complicates and increases the cost of treatment [1]. The development of accurate and simple methods to identify isolates of $S$. epidermidis and determine their methicillin resistance is important to establish the clinical relevance of $S$. epidermidis [6].

The emergence of $S$. epidermidis as an opportunistic pathogen is very critical. While anti-staphylococcal penicillins such as methicillin and oxacillin were considered as the first-line therapy options, the emergence of methicillin-resistant Staphylococci have impacted the concerns in healthcare units [7].

S. epidermidis on the skin may cause an opportunistic infection and also has the potential to become a reservoir of antimicrobial resistance genes; methicillin resistance gene $(\mathrm{mec} A)$. A previous study investigated the antimicrobial susceptibilities of $P$. acnes and $S$. epidermidis isolated from patients with acne vulgaris in a university hospital in Japan from 1996 to 1997. At that time, no antimicrobial-resistant strains were found for $P$. acnes, and $S$. epidermidis showed a high susceptibility to antimicrobials [3]. Methicillin-resistant staphylococcal strains acquired and integrated into their genome the staphylococcal cassette chromosome mec (SCCmec), which carries the methicillin resistance (mecA) gene encoding for an altered penicillin binding protein, and other antibiotic resistance determinants [8].

Resistance to methicillin was reported for the first time in 1961. In a study which was conducted by Guisti et al. in 1999, methicillin resistance ratio was reported (48.6\%). Resistance to mentioned antimicrobial groups has been even more increasing, for instance in Finland in 1983 the 28\% resistance was raised up to $77 \%$ in 1994 . Currently 75 up to $90 \%$ of $S$. epidermidis isolates from various nosocomial infections in most European and American countries are methicillin-resistant strains [7].

Concordant with the increment of coagulase-negative Staphylococci importance particularly $S$. epidermidis as an agent of nosocomial infections, further clinical and experimental studies in different fields such as mechanism of transmission, ecology, virulence factors, typing methods and antimicrobial resistance mechanism are needed. The prevention and treatment of $S$. epidermidis infections should depend on our knowledge about the reservoirs, epidemiology, and host defenses against these microorganisms [7]. Thus this paper aimed to present the identification of methicillin resistant Staphylococcus epidermidis in acne of patients with different levels of severity.

\section{Materials and Methods}

A total of 46 females who met the criteria of acne vulgaris with degree of acne ranging from mild, moderate and severe attending Wahidin Sudirohusodo hospital, Hasanuddin University hospital and among senior high school students in the city of Makassar were studied. The research was conducted by examining clinical manifestations and sebum levels. Specimens were obtained from comedonal lesions, papulopustular lesions, or nodular lesions at the facial area, the number of papul or pustul were counted and used to determine the classification of mild, moderate and severe acne based on combined acne severity classification. PCR technique was performed in order to detect the presence of Methicillin-resistant Staphylococcus epidermidis. Prior to sampling, the subjects had signed informed consent.

\subsection{Materials}

Primers used in this study were MRS1 (5'-TAG AAA TGA CTG AAC GTC CG-3') and MRS2 (5'-TTG CGA TCA ATG TTA CCG TAG-3'). Go taq Master Mix Green, Hot Star Taq DNA polymerase 0.5 TAE buffer, and 100 base pair DNA ladder were used. The PCR results were validated with a clinically relevant strain isolated from acne lession. The samples were taken each with sterile swabs placed in Phosphate Buffered Solution (PBS) and brought to the Microbiology Laboratory of Hasanuddin University Hospital. All samples were kept at $-20^{\circ} \mathrm{C}$ until ready for PCR examination.

\subsection{PCR Method}

DNA extraction from swab samples was performed according to the methods recommended by Geneaid. Initially S2 solution was prepared by adding $1 \mu \mathrm{l}$ RNA Carrier with $500 \mu 1 \mathrm{~S} 2$ buffer to each samples. Into the swab samples, 500 $\mu 1$ of S1 solution and $20 \mu$ Proteinase $\mathrm{K}$ was added, vortexed for ten seconds and incubated at $60^{\circ} \mathrm{C}$ for 10 minutes. Each sample were then transferred to column filter on $1.5 \mathrm{ml}$ microtube, sentrifuged at 14000-16000 (15000) X g for two minutes. The filter column was then removed. After adding $500 \mu 1$ of S2 solution, vortexed, incubated at $60^{\circ} \mathrm{C}$ for ten minutes including vortexing every 5 minutes, $500 \mu 1$ Ethanol Absolute was added and mixed for ten seconds. The solution was transferred $750 \mu \mathrm{l}$ into GD Column on $2 \mathrm{ml}$ collection tube, sentrifuged at 14000-16000 (15000) X g for one minute. This process was repeated once more, then the collection tube was discarded, and replaced with a new $2 \mathrm{ml}$ collection tube.

Washing: Buffer W1 400 ul was added into the GD column, centrifuged at 14000-16000 (15000) X g for 30 seconds, then liquid contained in the collection tube was discarded. After adding 600 ul Wash Buffer (previously was added $100 \mathrm{ml}$ Ethanol), tube was centrifuged at 14000-16000 (15000) X g for 30 seconds. The liquid contained in the collection tube was discarded and centrifuged again at a speed of 14000-16000 (15000) X g for 3 minutes.

Elution: the GD column was transferred on $1.5 \mathrm{ml}$ microtube, previously heated elution buffer was added $100 \mathrm{ul}$, and allowed to stand for 3 minutes before centrifuged at14000- 16000 (15000) X $\mathrm{g}$ for five minutes. The GD column was discarded and the liquid contained in the microtube contained the DNA ready to be tested for PCR.

\subsection{Amplification}

The amplification was performed on a Thermal Cycler 
(Mastercycler, Eppendorf, Hamburg, Germany) using $25 \mu 1$ of PCR mixture containing $12.5 \mu \mathrm{l}$ of master mix green, 0.5 $\mu \mathrm{l}$ of Forward Primer, $0.5 \mu \mathrm{l}$ of Reverse Primer, $6.5 \mu \mathrm{l}$ Nuclease Free Water, and $5 \mu \mathrm{l}$ DNA product. Amplification were performed under these conditions: denaturation for 3 min at $94^{\circ} \mathrm{C}$, followed by 30 cycles of $94^{\circ} \mathrm{C}$ for $1 \mathrm{~min}, 58^{\circ} \mathrm{C}$ for $1 \mathrm{~min}$ and $72^{\circ} \mathrm{C}$ for $1 \mathrm{~min}$, with final extension at $72^{\circ} \mathrm{C}$ for 5 minutes. Amplified products were analyzed using $1.5 \%$ of agarose gel electrophoresis with GelRed 1X (Biotium, California, USA), visualized and captured on UV transilluminator (Mini BIS Pro, DNR Bio Imaging Systems, Jerusalem, Israel).

\section{Results}

Table 1. PCR Results on 46 acne samples at various level of severity.

\begin{tabular}{llll}
\hline \multirow{2}{*}{ Degree of Acne } & MRSE & & Total \\
\cline { 2 - 4 } & + & $6(13,0)$ & 15 \\
Mild & $9(19,6)$ & $6(13,0)$ & 16 \\
Moderate & $10(21,7)$ & $6(13,0)$ & 15 \\
Severe & $9(19,6)$ & $18(39,0)$ & 46 \\
Total & $28(61,0)$ & $X^{2}$ & \\
\hline
\end{tabular}

From the table above we can see that more than half of the study subjects with acne $(61,0 \%)$ were infected with $S$. epidermidis which were MRSE. It is consistent with the theory that acne vulgaris patient should not be given MRSE class antibiotics because they contained bacteria which are already resistant. However the positivity of MRSE on different severity of acne did not differ significantly ( $p>0.05$ ), meaning that in all patients with acne vulgaris regardless of its degree of severity, should not be given antibiotics of the MRSE class.

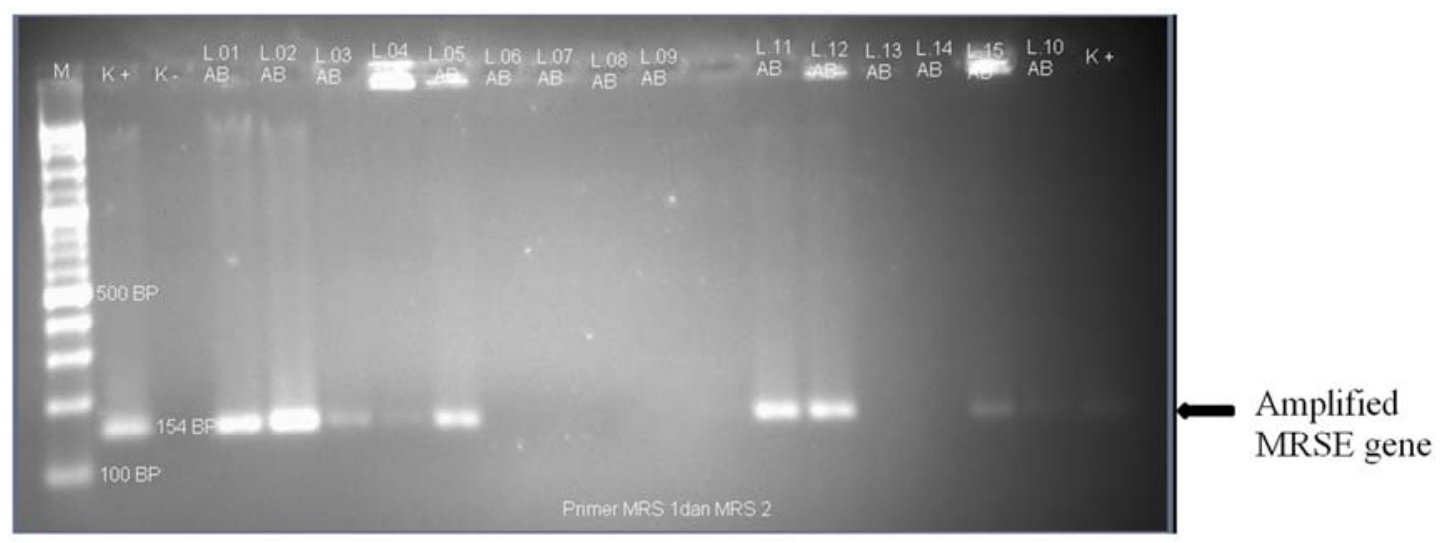

Figure 1. Methicillin-resistant Staphylococcus epidermidis PCR result among severe acne group.

Figure 1 showed that there were 9 positive samples and 6 negative samples in MRSE PCR among samples with severe acne.

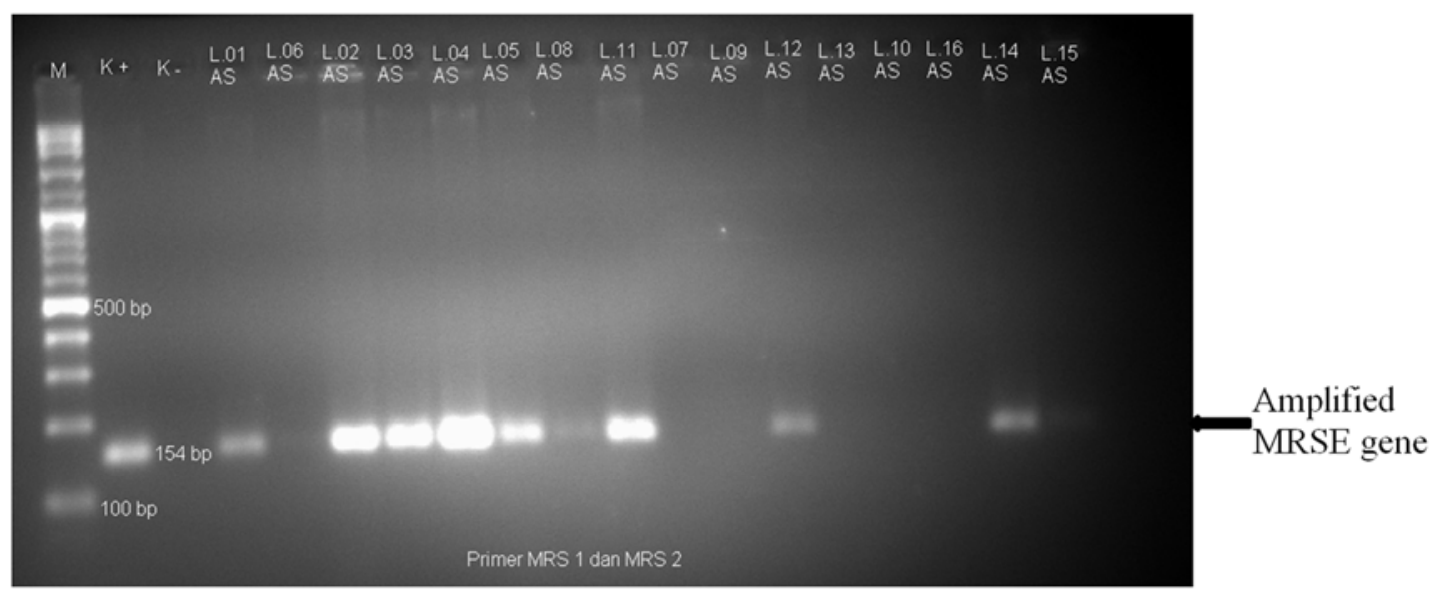

Figure 2. Methicillin-resistant Staphylococcus epidermidis PCR result among moderate acne group.

Figure 2 showed that there were 10 positive samples and 6 negative samples in MRSE PCR among moderate acne group. 


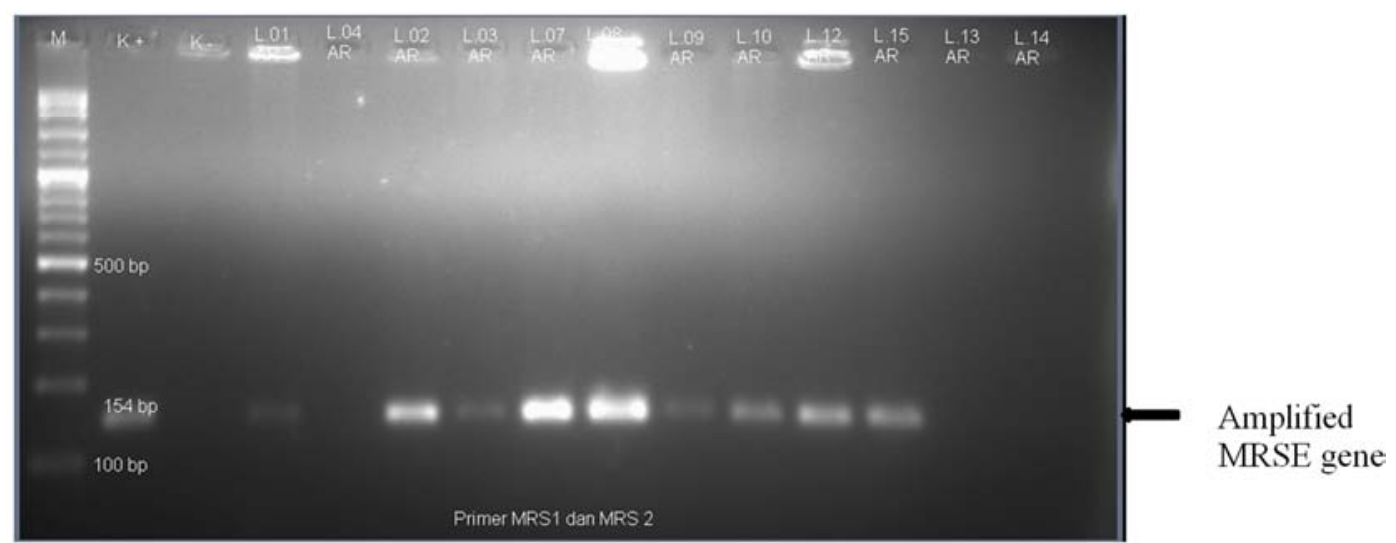

Figure 3. Methicillin-resistant Staphylococcus epidermidis PCR result among mild acne group

Figure 3 showed that there are 9 positive samples and 6 negative samples in MRSE PCR result in mild acne group.

\section{Discussion}

Staphylococcus epidermidis (S. epidermidis) is a common commensal bacterium of the human skin and mucosa. While S. epidermidis has long been considered nonpathogenic, it is now recognized as a relevant opportunistic pathogen [9].

Understanding the patterns of resistance and tolerance of $S$. epidermidis to antimicrobial agents is essential for the appropriate diagnosis and management of $S$. epidermidis infections. Antibiotic resistance patterns might be useful in differentiating a nosocomial isolate from a communityacquired isolate. S. epidermidis is one of the leading nosocomial organisms with a characteristic multidrugresistant pattern, including resistance to methicillin [10].

The increasing antibiotic resistance of nosocomial isolates of $S$. epidermidis poses a great challenge for the management of hospital-acquired infections in general. Worldwide surveillance shows that the isolation frequency of methicillinresistant $S$. epidermidis (MRSE) can reach $80 \%$ in hospitals [2].

$S$. epidermidis is one of the normal inhabitants of the skin and easily acquires and transmits the various antimicrobial resistance genes [3]. Methicillin resistance is frequently found also in $S$. epidermidis, and may originally have been transferred to $S$. aureus from this species [1].

Molecular factors that determine staphylococcal pathogenesis have been extensively investigated. Aggressive virulence determinants such as toxins are mostly found in $S$. aureus, while other species mostly lack the production of toxins. Interestingly, in $S$. epidermidis as the most intensively studied species other than $S$. aureus, for the most part, factors that have been implicated in pathogenesis appear to have original functions in the noninfectious lifestyle of this bacterium. This indicates that these less aggressive species have not evolved to become pathogenic, but infection has to be regarded as an 'accident' rather than a program [11].

The accidental nature of infection with species such as $S$. epidermidis suggests that the frequency of infections is to a large part determined by the abundance of these species on the human body, from where infection is believed to originate. The most important sources of infection with $S$. epidermidis and many other staphylococci are likely the skin and mucous membranes of patients or healthcare personnel [11].

This study found $61.0 \%$ MRSE resistance in acne infected with staphylococcus epidermidis. Although the history of antibiotic use was not known among these patients, the prevalence of those antimicrobial resistance genes in $S$. epidermidis may be caused by the previous use of clindamycin and gentamicin in treating skin and soft tissue infections. The pathogenicity of $S$. epidermidis for acne vulgaris remains unclear and is assumed that $S$. epidermidis could become a reservoir of various antimicrobial resistance genes on the skin [3].

Based on a suggestion from a study by Keisuke et al, the existence of antimicrobial-resistant bacteria should be considered when selecting antimicrobial therapy for inflammatory acne, and antimicrobials should not be used for the maintenance therapy of acne vulgaris [3]. This suggestion is appropriate with the finding in our study.

\section{Conclusion}

Simple PCR identified MRSE from pus speciment of acne patients at various levels of severity, however the level of severity of acne has no significant correlation with the presence of MRSE. Acne vulgaris patient should not be given MRSE class antibiotics because they contained bacteria which are already resistant, meaning that all patients with acne vulgaris regardless of its degree of severity, should not be given antibiotics for maintenance therapy.

\section{References}

[1] Otto M. Staphylococcus Colonization Of The Skin And Antimicrobial Peptides. NCBI. 2010; 5 (2): 183-95.

[2] Watanabe K, Nakaminami H, Azuma C, Tanaka I, Nakase K, Matsunaga N, et al. Methicillin-Resistant Staphylococcus Epidermidis Is Part Of The Skin Flora On The Hands Of Both Healthy Individuals And Hospital Workers. Biol Pharm Bull. 2016; 39: 1868-75. 
[3] Nakase K, Nakaminami H, Takenaka Y, Hayashi N, Kawashima M, Noguchi N. Relationship Between The Severity Of Acne Vulgaris And Antimicrobial Resistance Of Bacteria Isolated From Acne Lesions In A Hospital In Japan. Journal of Medical Microbiology. 2014; 63: 721-8.

[4] Hassanzadeh P, Bahmani M, Mehrabani D. Bacterial Resistance To Antibiotics In Acne Vulgaris: An In Vitro Study. Indian J Dermatol. 2008; 53: 122-4.

[5] Wang Y, Kuo S, Shu M, Yu J, Huang S, Dai A, et al. Staphylococcus Epidermidis In The Human Skin Microbiome Mediates Fermentation To Inhibit The Growth Of Propionibacterium Acnes: Implications Of Probiotics In Acne Vulgaris. Appl Microbiol Biotechnol. 2014; 98.

[6] Iorio NLP, Azevedo MB, Frazão VH, Barcellos AG, Barros EM, Pereira EM, et al. Methicillin-Resistant Staphylococcus Epidermidis Carrying Biofilm Formation Genes: Detection Of Clinical Isolates By Multiplex PCR. Int Microbiol. 2011; 14: $13-7$.
[7] Namvar AE, Bastarahang S, Abbasi N, Ghehi GS, Farhadbakhtiarian S, Arezi P, et al. Clinical Characteristics Of Staphylococcus Epidermidis: A Systematic Review. GMS. 2013; 9 (3): 1-10.

[8] Ibrahem S, Salmenlinna S, Lyytikäinen O, Vaara M, VuopioVarkila J. Molecular Characterization Of Methicillin-Resistant Staphylococcus Epidermidis Strains From Bacteraemic Patients. Clin Microbiol Infect. 2008; 14: 1020-7.

[9] Cherifi S, Byl B, Deplano A, Nonhoff C, Denis O, Hallind M. Comparative Epidemiology Of Staphylococcus Epidermidis Isolates From Patients With Catheter-Related Bacteremia And From Healthy Volunteers. JCM. 2013; 51: 1541-7.

[10] Raad I, Alrahwan A, Rolston K. Staphylococcus Epidermidis: Emerging Resistance And Need For Alternative Agents. Clin Infect Dis. 1998; 26: 1182-7.

[11] Otto M. Staphylococcus Epidermidis - The 'Accidental' Pathogen.. Nat Rev Microbiol. 2009; 7: 555-67. 